\title{
Le dopage entre performance et perception du corps en Espagne
}

\author{
Noemi García-Arjona et Teresa González-Aja \\ Facultad de Ciencias de la Actividad Física y del Deporte-INEF Universidad Politécnica de Madrid, C/Martín Fiero 7, \\ 28040 Madrid, Espagne
}

\begin{abstract}
Reçu le 16 février 2015 - Accepté le 18 mars 2015
Résumé. La nécessité d'aborder un sujet aussi complexe comme le dopage est donné par une préoccupation pas seulement dans le monde du sport, mais aussi dans l'espace public. Des nombreux exemples montrent le croissant intérêt scientifique et une plus grande couverture médiatique. Cependant, on trouve exceptionnellement des études rigoureuses dans une perspective historique. Moins nombreux encore on peut trouver des exemples dans le contexte espagnol. Le but de ce texte est d'offrir une approche analytique de la histoire du dopage en Espagne. Sous l'exemple des affaires de dopage des sportifs espagnols apparus dans les journaux sportifs on va définir les éléments pour établir l'analyse avec une rigueur historique et objective et remettre en question la vision d'un seul sport «pur » et «bon».
\end{abstract}

Mots clés : Sport, dopage, histoire, Espagne, journaux sportifs

\begin{abstract}
The doping between performance and perception of the body in Spain.
The need to address such a complex issue as doping is given by a concern, not only in the sport context, but also in the public arena. Numerous examples illustrate the growing scientific interest and greater media coverage. However, there are few studies with deep and rigorous treatment of historical perspective. Even fewer examples can be found in the Spanish context. The purpose of this text is to offer an analytical approach to the history of doping in Spain. The example of doping cases of Spanish athletes published in the sports newspapers will be used to define the evidences for establishing the analysis with historical and objective rigor and to question the vision of a "pure" and "good" sport.
\end{abstract}

Key words: Sport, doping, history, Spain, sport newspapers

\section{Introduction}

\subsection{Construction d'un corpus sur le dopage en Espagne : la presse sportive}

Pour l'étude du contexte espagnol, il a été nécessaire de délimiter deux périodes pour chercher à connaître l'impact de la première législation antidopage en Espagne. La méthodologie empruntée consista donc à partir des grandes affaires dont la proximité temporelle avec une transformation de la législation au niveau du dopage est suffisamment forte pour que l'on puisse supposer un mouvement dans l'opinion à l'origine de ce changement. Comme l'affirment Dimeo (2007) et Møller (2012), la politique antidopage agira comme un révulsif sur le discours social, et d'abord, sur le discours des médias, pour les affaires de dopage. D'ailleurs, l'opposition presque universelle à l'usage de substances n'existait pas jusqu'au début des années 1960, années où le dopage a commencé à être considéré comme un risque pour la santé, plus que par éthique sportive. Sur la base de ces faits historiques et pour le contexte espagnol, nous proposons les phases suivantes :

- Première Période : 1975-1990, de la mort de Franco et de la Transition démocratique jusqu'à la première législation espagnole contre le dopage, incluse dans la Loi 10/1990, du 15 octobre, sur le sport.

- Deuxième Période : 1991-2010, de la création de la Commission antidopage en Espagne à travers le Conseil supérieur des sports espagnol jusqu'à nous jours.

Le poids de la presse espagnole commence à se consolider lors de la première période. Elle se développe pendant une époque déterminante, la Transition : créatrice d'opinion publique mais aussi facteur principal actif du fonctionnement démocratique. Ce sont pendant ces premières années que s'est érigé le mouvement d'une presse indépendante, moderne et européenne qui devra relever le défi de lutter contre le poids de la dictature et sa 
claire oppression à la liberté d'opinion. Un exemple manifeste est la création de nouveaux journaux tels que $E l$ País en 1976, qui rassemblaient ce désir collectif de régénération et de démocratie médiatique (Chartier, 2011). En parallèle, il en sera de même avec la presse sportive. Contrairement à d'autres pays européens, la présence et l'importance des médias sportifs spécialisés n'était pas un phénomène nouveau ni isolé en Espagne ${ }^{1}$ bien que la pratique sportive dans l'ensemble de la société était encore faible (González Aja, 2011, p. 323-353) La Catalogne se construisait, depuis le début du siècle, comme la région la plus active et avec une tradition plus ancienne dans des publications sportives jusqu'à ce moment (Pujadas i Martí \& Santacana, 1997) comme par exemple le journal Mundo Deportivo, fondé en 1906. Mais ce ne sera que dans les années 1980 que le phénomène populaire d'expansion médiatique se produira réellement, tel que nous le connaissons à l'heure actuelle. En ce sens, López López (2011) différencie trois époques d'évolution : le tardo-franquisme et la transition, « une époque dorée » née des réussites sportives du moment, l'effet des Jeux Olympiques de Barcelone 92 dans les années 1990, et une troisième période marquée par l'incorporation du format numérique et la consolidation de journaux sportifs en expansion, devant même des quotidiens d'information générale.

Dans le cas de l'Espagne, deux des plus grands journaux sportifs (Marca et Mundo Deportivo) font l'objet d'une étude pendant la période historique qui nous concerne. Sous le régime de Franco, la censure de la presse était une activité quotidienne dans tout ce qui portait atteinte aux valeurs du franquisme, le sport «pur», l'image et la fierté de la «race espagnole» (Viuda Serrano, 2010). Cette censure s'est petit à petit réduite pendant les dernières années de la dictature, accompagnée d'une certaine politique d'ouverture dans tous les domaines de la société, l'information et la culture, qui a coïncidé avec une étape d'amélioration économique naissante.

Pour leur éditorial et leur situation géographique dans deux grandes villes espagnoles, aussi rivales sur le plan politique que sportif (Marca à Madrid, Mundo Deportivo à Barcelone), la comparaison de ces deux publications nous permet d'obtenir un aperçu précis sur les sports en Espagne.

Le quotidien Marca est né pendant la Guerre Civile (le 21 décembre 1938). C'est actuellement le journal le plus lu en Espagne, devant même des journaux d'information générale ${ }^{2}$ Dans le graphique suivant, nous pouvons observer cette différence quantitative : avec 2870000 lec-

1 López López insiste sur le fait que l'hégémonie de la presse sportive est une caractéristique des pays d'Europe du Sud comme l'Espagne, l'Italie ou le Portugal (López López, 2011, p. 393-432).

2 D'après les données recueillies par l'EGM (Étude générale de médias) pendant la période d'octobre à mai 2013. http:// www.aimc.es//-Datos-EGM-Resumen-General-.html consultée le 14-11-2013.

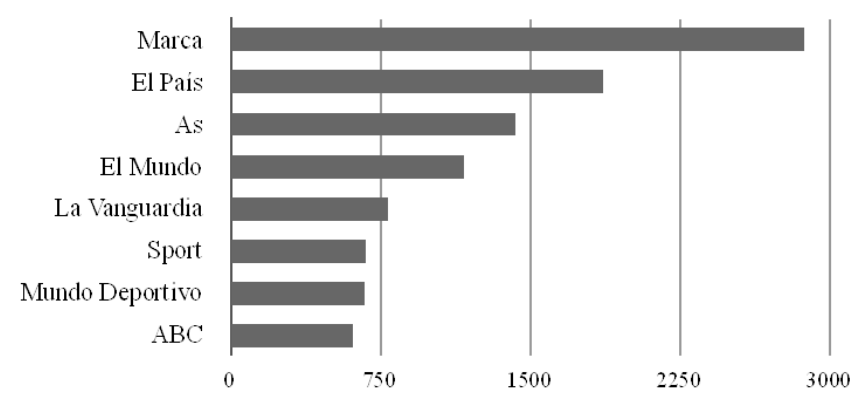

Fig. 1. D'après les données recueillies par l'EGM (Étude générale de médias) pendant la période d'octobre à mai 2013 (http://www.aimc.es//-Datos-EGM-ResumenGeneral-.html consultée le 14-11-2013).

teurs par jour, ce journal sportif devance un million lecteurs El País (1862000 lecteurs par jour) et le nombre double avec le journal sportif $A s$, le troisième le plus lu d'Espagne (Fig. 1).

Dans le cas de Mundo Deportivo, ce quotidien a été publié à Barcelone pour la première fois en 1906, garantissant le sport en tant que phénomène social avec sa seule présence ininterrompue. C'est le journal sportif le plus vieux d'Espagne et le deuxième d'Europe, derrière l'italien Gazzetta dello Sport, fondé en 1896.

La presse spécialisée va refléter, par conséquent, l'audience du phénomène sportif dans le pays, ce qui permet l'accès à une vision plus vaste de l'avancée d'un nouveau phénomène : la dénonciation d'un comportement contraire au «franc-jeu» et non au «sport ». Le sport se constitue comme véritable «phénomène social» pour que le public des lecteurs se l'approprie, en même temps que les attributions juridiques du quotidien sportif sont introduites dans le même processus.

\subsection{Justification du choix des affaires de dopage analysées et indicateurs pour leur interprétation}

Le choix d'une analyse parallèle de deux quotidiens spécialisés nous permet de récupérer le discours prédominant du sport par le biais des journalistes et de chroniqueurs influents. Nous utilisons une méthode de comparaison systématique afin d'identifier des nouvelles qui mentionnent des faits relatifs à l'utilisation de substances dopantes. Pour cela, nous nous appuyons sur l'analyse que proposent Perera et Gleyse (2005) en prenant comme référence le traitement du discours des journalistes sportifs quant à la dualité de pur et impur : d'une part, le discours de la pureté dans le sport et en général, dans la pratique physique-sportive, le mens sana in corpore sano, de la noblesse sportive sans discussion, voire de la primauté d'une identité nationale, pour le bien de la santé mentale et physique du sportif. Sous cette prémisse, seulement l'autorité médicale apparaît comme l'unique capable de délimiter cette pratique. D'autre part, l'impur, celui qui tombe dans les 《réseaux» du dopage est stigmatisé et 
condamné bien que peu de jours avant il eût été porté aux nues par les mêmes journalistes. En effet, il est important de retenir le rôle du journaliste dans le processus de formulation des nouvelles sur le dopage. Il peut, de son plein gré ou à son regret, se transformer en « juge » de l'affaire qu'il aborde, en rendant tel ou tel jugement à travers son discours et à travers le journal. Ainsi, le panorama des différentes positions possibles face au «crime» se dessine peu à peu et les nuances qui en émanent permettent de comprendre la construction de l'opinion publique produite. Nous avons privilégié les indicateurs suivants dans l'analyse des nouvelles pour répondre en profondeur aux blocs thématiques envisagés :

- la moralité et la légalité dans les affaires de dopage,

- la nationalité (comme espace sportif d'exaltation du patriotisme, l'identité),

- le rôle du journaliste en tant que juge,

- les différences entre les sports : cyclisme, basketball et athlétisme,

- la question de genre et d'âge (athlète perçu comme « éternel», surhomme).

Pour la sélection des affaires de dopage des deux journaux, nous avons pris en considération une série de critères analogues non pas tant au niveau de la représentativité statistique (sport, genre, impact médiatique) mais plutôt au niveau de son importance par rapport aux indicateurs thématiques d'analyse que nous proposons dans le présent rapport, c'est-à-dire : 1) relation entre corps et dopage, 2) raisons pour se doper ou non et 3) prévention contre le dopage. Il est important de souligner le fait qu'en basketball, nous n'avons trouvé aucune affaire de sportifs espagnols qui ait vu publiquement le jour, mais en revanche nous avons trouvé des informations sur la politique antidopage appliquée, fait dont nous rendrons compte tout au long de l'étude. De cette manière, les affaires prises pour référence sont les suivantes:

- Période I (1975-1990) : Sebastián Pozo (Tour de France 1977), Bartolomé Caldentey (championnat du monde de cyclisme amateur 1982), Ángel Arroyo (Tour d'Espagne 1982), Pedro Delgado (Tour de France 1988) et Cristina Pérez (athlétisme, JO de Séoul 1988).

- Période II (1991-2010) : Joan Llaneras (championnat du monde de cyclisme sur piste 2001), Alberto García (championnat du monde de Cross à Lausanne 2003), Opération Puerto (2006), Maribel Moreno (cyclisme, JO de Pékin 2008), Opération Grial (2009), Marga Fullana (cyclisme BMX, 2010), Alberto Contador (Tour de France 2010) et Opération Galgo (2010).

\section{Rendement, corps et dopage : loi du silence ou ingénuité (in)consciente}

Dans l'analyse des nouvelles sur le dopage en Espagne des deux périodes historiques (de 1975 à 1990 et de 1990 à aujourd'hui), nous trouvons peu de références à la relation établie par que la nouvelle de l'athlète protagoniste d'une affaire de dopage ou suspecté de dopage et son rendement sportif ou perception de son corps. Dans peu de cas, cette nouvelle approfondit les effets que recherche l'athlète en se dopant pour améliorer son rendement. Surtout parce que, sauf des cas isolés, il nie toujours le contrôle positif exposé au grand jour.

De fait, nous pouvons observer que les premières chroniques sont accompagnées d'une interview de l'athlète, son entraîneur ou de son porte-parole, mais ce dialogue entre la personne touchée et la presse accomplit dans l'ensemble des affaires analysées prend les mêmes contours : une possibilité d'explication et de défense envers l'opinion publique ainsi qu'une négation de l'acte de dopage.

En ce sens, nous pouvons dire que quand nous prenons en compte l'une ou l'autre période historique, les cas de dopage qui voient le jour ont une couverture médiatique en fonction de l'envergure internationale de l'athlète et de sa place de star sportive de premier rang. Autant indiquer que le niveau de compétition intervient ici puisque les contrôles sont réalisés davantage parmi l'élite, c'est-àdire, par rapport à des sportifs et des sportives qui ont des objectifs de rendement particulièrement élevés.

Par exemple, dans la première période (1975-1990), sont seulement apparues des affaires concernant, pour la plupart, le cyclisme; un cas est seulement apparu aux Jeux de Séoul 88 dans une autre APS. Un cas en athlétisme est dévoilé à cette occasion, mais dans le cadre d'une discipline minoritaire qu'est le pentathlon, avec l'affaire Quesada avait, alors, obtenu une modeste $24^{\mathrm{e}}$ place dans le classement final. D'après Mundo Deportivo, du propranolol a été trouvé dans l'urine de Quesada. Il s'agit d'un amortisseur des effets de l'adrénaline, pour calmer la nervosité. Ce bétabloquant de première génération peut donc avec profit être utilisé chez les tireurs sportifs. Quesada déclara qu' «il avait seulement pris des herbes et que celles-ci n'avaient jamais provoqué un contrôle positif ${ }^{3} »$ bien qu'ensuite il reconnut son contrôle positif par lettre et fut déclaré par le président du COE au moment de ces jeux, Ferrer Salat, comme « entièrement coupable », pour finalement être sanctionné par la fédération de pentathlon tant espagnole qu'internationale.

Que ce soit en cyclisme ou en athlétisme, le traitement de la presse sur les affaires est très naïf ou consciemment couvert. La relation de son entourage le plus proche, que ce soit son l'équipe technique, les parents ou les suiveurs, est toujours décrite de façon unanime par la presse comme une défense à outrance du sportif, sur lequel une injustice a été commise; parfois, la présentation relève d'une logique victimaire encore plus approfondie : la sportive ou le sportif est déclaré alors victime d'un véritable complot.

Un exemple de cette défense tenace de la part d'un entraîneur ou d'une équipe technique de l'innocence de l'athlète inculpé est observé dans le cas du cycliste Ángel

3 Vives L., «El doping salpica a España », Mundo Deportivo, 25/09/1988, p. 42. 
Arroyo dans le Tour d'Espagne de 1982. En mai 1982, le cycliste, star de l'équipe Reynolds, gagne le Tour d'Espagne mais à peine trois jours après la nouvelle éclate : Arroyo a été contrôlé positif dans l'étape de montagne qui a eu lieu à Navacerrada (Madrid), avec la disqualification qui en découle. C'est par la presse, concrètement par un journaliste de Mundo Deportivo, Javier Dalmases, que le cycliste apprend la nouvelle du contrôle positif $^{4}$. La contre-analyse réalisée à l'Institut toxicologique de l'INEF confirme le résultat et les réactions ne tardent pas à apparaître : indignation, surprise, injustice, etc. Les chroniques de défense du cyclisme espagnol ne se font pas attendre, comme celle de Dalmases le jour suivant $^{5}$, dans lesquelles sont aussi inculpées les équipes technique et médicale dans leur responsabilité des cas de dopage, y compris en proposant une seconde lecture des substances dopantes surtout celles qui en petite quantité n'améliorent pas le rendement sportif mais sont utilisées pour traiter des maladies communes.

Dans le cas d'Arroyo, nous pouvons observer comment le niveau de rendement ou de catégorie du sportif influence la manière dont l'équipe ou l'entourage va le soutenir. Arroyo étant la figure sportive nationale par excellence - puisqu'il est le nouveau gagnant de la patrie de l'épreuve cycliste la plus importante d'Espagne, le Tour d'Espagne (Vuelta ciclista a España en espagnol) -, va jouir d'un traitement inégal en sa faveur au sein de l'équipe Reynolds par rapport à ses coéquipiers. De fait, même s'il existait une clause dans le contrat du cycliste selon laquelle son contrat serait résilié s'il était contrôlé positif à un test de dopage, il n'en fut rien et l'équipe Reynolds défendit le cycliste jusqu'à la fin, y compris quand le contrôle positif fut confirmé et Arroyo perdit le titre. Au contraire, il arriva la même chose à un autre coureur de la même équipe, Juan María Azcárate, l'année précédente (1981) dans une épreuve cycliste de second rang, la Clásica de Saint Sébastien, et effectivement, son contrat fut immédiatement annulé pour avoir été contrôlé positif $^{6}$. La condition de «domestique» et non de chef de files au sein de l'équipe fut fondamentale pour déterminer la position de l'équipe technique face au scandale du contrôle positif.

Toutefois, au sein de l'équipe, on doit souligner la manière dont le sportif impliqué, Arroyo, leader et vainqueur du Tour, se présente par rapport à ses propres collègues. Quand la nouvelle de son contrôle positif éclate, les premières déclarations d'Arroyo se dirigent vers sa propre équipe :

«Ce qui me gène le plus, termine le cycliste, c'est que ce qui m'a coûté autant d'efforts, et non seulement à moi mais aussi à toute l'équipe "Reynolds",

4 Dalmases J., « Arroyo se enteró por nosotros. 'Se me ha parado el corazón' », Mundo Deportivo, 12/05/1982, p. 30.

5 Dalmases J., « Los ciclistas no son drogadictos », Mundo Deportivo, 13/05/1982, p. 33.

${ }^{6}$ Carrasco J., « Reynolds sigue dudando de la veracidad del positivo », Marca, 15/05/1982, p. 19. maintenant, s'est envolé en un seul jour comme ça. Je suis désolé surtout pour mes coéquipiers qui ont lutté autant voire plus que moi et finalement cela n'a servi à rien ${ }^{7} \gg$.

La question de la concurrence entre sportifs n'influence pas a priori la position face à l'affaire de dopage. De plus, parmi les cas analysés, les rivaux directs se positionnent clairement en faveur du sportif inculpé, en signe de solidarité et de respect pour les mérites obtenus et en dénonciation contre le système de dopage qui l'a «injustement » dépossédé de ses titres.

Mais nous pouvons trouver ici des différences entre certains sports. Dans les cas où les cyclistes sont impliqués, ils se soutiennent et se protègent entre eux. Cependant, dans le cas de Cristina Pérez, en athlétisme, la position est très différente. L'athlète est exclue, de manière privée par le groupe des autres athlètes ou bien de manière publique par les médias. Même étant disculpée du positif en raison d'erreurs dans le contrôle positif, la mauvaise réputation l'accompagnera durant toute sa carrière sportive.

Dans les exemples de solidarité entre collègues cyclistes, le premier à souligner est celui du cycliste Arroyo. Pour son contrôle positif, il a finalement dû céder son titre de champion du Tour d'Espagne à Mariano Lejarreta, qui affirmait, convaincu, qu'il ne se sentait pas fier du titre puisqu'il considérait Arroyo « vainqueur moral » et, par conséquent, que cette victoire «n'a pas le goût de la gloire $^{8} »$. Le second cas est celui de Pedro ou « Perico» Delgado pendant le Tour de France de 1988. Le chef de files de l'équipe Reynolds se trouvait en première position au classement général du Tour de France et était un candidat important pour répéter le geste que feront d'autres Espagnols comme Bahamontes (1959) et Ocaña (1973). Perico a été contrôlé positif le 19 juillet 1988 au probenecid, une substance qui permet d'inhiber l'excrétion rénale de certaines drogues et qui, par conséquent, pouvait agir comme agent pour masquer des substances interdites. Le positif a été confirmé au jour suivant avec la contre-analyse mais fut finalement annulé et Delgado gagna le Tour, car cette substance faisait partie de la liste des substances interdites du CIO et donc, du Tour de France, mais non pas de l'UCI (Union cycliste internationale). Le deuxième au classement général, Steven Rooks, cycliste néerlandais leader de l'équipe PDM, s'est clairement positionné même avant le verdict final et rejeta la possibilité d'obtenir le maillot jaune, en affirmant que « le gagnant de ce Tour est, sans aucun doute, lui $[\text { Pedro Delgado }]^{9} »$. Cette légitimité du vainqueur malgré son contrôle positif rend suspecte la défense même d'un sport sans dopage. Tout se passe comme si le second au

\footnotetext{
7 Dalmases J., «Arroyo : 'pese a todo, yo soy vencedor de la Vuelta'», Mundo Deportivo, 15/05/1982, p. 27.

8 Sagastume I., « Marino Lejarreta, ganador de la Vuelta de rebote », Marca, 15/05/1982, p. 19.

9 Harrie Jansen, patron de PDM, muy claro : «Rocks no quiere el amarillo ». Marca, 21/07/1988, p. 4.
} 
classement validait le résultat confirmant son niveau inférieur, et par conséquent validait du même coup le statut supérieur du vainqueur, pourtant accusé, puis convaincu, de dopage. Cette manière de faire laisse entendre que la hiérarchie avec dopage est la véritable hiérarchie sportive. Ce type d'attitudes ne peut pas ne pas avoir d'incidences sur les spectateurs et téléspectateurs de ces feuilletons sportifs et judiciaires en lien avec les affaires de dopage. . .

Un cas atypique dans les relations entre collègues sportifs et l'implication dans une affaire de dopage, est trouvé avec l'athlète Cristina Pérez en 1988. Pérez était une jeune athlète prometteuse, spécialiste dans les épreuves de vitesse et recordwoman depuis 1987, ainsi que demi-finaliste à Séoul 1988 aux 400 mètres haies. Elle fut contrôlée positive au Meeting Ciutat de Barcelona, en juillet 1988, au chlorphentermine, un agent amaigrissant interdit par l'IAAF. Cependant, la nouvelle du contrôle positif ne s'est faite connaître qu'une fois passés les JO de Séoul 1988 puisque le président de la Fédération Espagnole d'Athlétisme, Juan Manuel de la Hoz, dissimula l'affaire pour que Pérez puisse participer aux Jeux Olympiques $^{10}$. L'importance médiatique de l'affaire et la relation sentimentale de Pérez avec le docteur Eufemiano Fuentes - médecin du sport directement impliqué dans les récentes affaires de dopage en Espagne (opération Puerto et opération Galgo) et déjà connu tant dans les milieux sportifs que journalistiques pour les méthodes suspectes qu'il utilisait pour améliorer le rendement chez les athlètes - ont marqué, d'après la compagne affectée, une campagne intentionnelle de discrédit. Ainsi, Cristina Pérez se faisait appeler communément dans le milieu de l'athlétisme «la pharmacie ${ }^{11} 》$ et par les médias euxmêmes : « la pharmacie ambulante s'est qualifiée pour les demi-finales ${ }^{12} \gg$. Deux années après l'affaire, le contrôle positif ayant finalement été invalidé du fait des irrégularités dans le contrôle ${ }^{13,14}$ et ayant été l'athlète elle-même celle qui passa volontairement le contrôle pour autoriser le record obtenu au Meeting de Barcelone. Pour autant, Pérez trouva peu de soutien parmi les autres athlètes, comme le dénonça deux ans après dans une interview à Mundo Deportivo :

«J'ai battu deux records en juillet, aux 100 et 400 mètres haies. Quelques mois plus tard, il a été dit que j'avais été contrôlée positive à un contrôle dont j'ai plaidé qu'il ne s'était pas réalisé de façon réglementaire. On $m$ 'a retiré le record aux 100 mètres, et celui des 400 mètres haies je l'ai,

\footnotetext{
10 Tarin Alonso M. « Expediente a de Hoz por el 'doping' de Cristina Pérez », Mundo Deportivo, 13/10/1988, p. 38.

11 Christina Perez acudió a Seúl pese al positivo de Barcelona, Marca, 21/07/1988, p. 20.

12 Vives L., «Cristina Pérez : 'triunfar en España es sinónimo de doparse'», Mundo Deportivo, 9/10/1988, p. 38.

13 Vives L. «El doping de Cristina, invalidado », Mundo Deportivo, $23 / 10 / 1988$, p. 43.

14 Vives L., «Las muestras, en botellas de Font Vella», Mundo Deportivo, 14/10/1988, p. 32.
}

car je l'avais battu plusieurs fois. Tout fut très étrange et presque personne ne s'est préoccupé de mon moral ${ }^{15} \gg$.

La preuve définitive de ce manque de soutien dans l'entourage de l'athlète s'est matérialisé par le renoncement de son entraîneur, Manuel Pascua Piqueras, à continuer de l'entraîner, et elle décida de se retirer de l'épreuve de relais de $4 \times 400$ au championnat d'Europe de Split en 1990 auquel, pour une prétendue lésion, elle décida de ne pas participer motu proprio avec le reste de l'équipe, ce qui a provoqué un profond malaise chez ses coéquipières $^{16}$.

Dans la seconde période, à partir de 1990, la couverture médiatique est différente en ce qui concerne les affaires de dopage et les objectifs sportifs obtenus; les journaux vont rendre compte de chacune des affaires, indépendamment des objectifs de rendement de l'athlète objet de soupçons. Que ce soit pour les grandes stars sportives comme Alberto Contador en cyclisme, où bien d'athlètes dans des disciplines les plus minoritaires ou des catégories nationales et régionales, l'affaire fera l'objet d'une information de plus en plus rigoureuse et explicite par le journal bien qu'évidemment donnant une plus grande couverture selon la répercussion du sportif. Mundo Deportivo le fera de façon beaucoup plus intense à échelle régionale dans ses éditions du Pays Basque avec le cyclisme qui est un des sports les plus suivis au niveau populaire et qui jouit d'un vivier de figures locales importantes. D'ailleurs, les sportifs convaincus et/ou accusés de dopage peuvent plus tard utiliser le chantage auprès des médias, en vantant leur connaissance parfaite du milieu du dopage. C'est ce que fit C. Perez plus de 20 ans plus tard lorsque son mari se trouvait inextricablement mêlé à des poursuites judiciaires aux ramifications complexes ${ }^{17}$.

\section{Lésions, souffrance et récupérations rapides. . un « miracle » appelé dopage?}

Dans le contexte espagnol, la presse a reflété l'ambiguïté que les sportifs espagnols ont parfois montrée en ce qui concerne l'utilisation de substances dopantes pour, prétendument, surmonter une indisposition physique ou une maladie, ce discours étant une constante dans tous les cas analysés. Dans la première étape, surtout pendant les années 1970, le terme de «miracle » s'utilisait beaucoup quand un athlète ou un coureur se remettait d'une lésion évidente de façon héroïque, et non moins suspecte, et parvenait à gagner l'épreuve sportive le jour suivant.

\footnotetext{
15 Vives L. «Cristina Pérez se queja », Mundo Deportivo, 7/02/1990, p. 46.

16 Vives L. « Cristina Pérez, sin 'coach' », Mundo Deportivo, 18/09/1990, p. 52.

17 http://www.liberation.fr/sports/2008/11/19/cristinaperez-une-menace-pour-le-sport-espagnol 258095 (page consultée le 13/12/2013).
} 
Ainsi, le cycliste Agustín Tamames, futur gagnant du Tour d'Espagne en avril 1975, est interviewé au sujet de son retour à une équipe espagnole, le Súper Ser (avec Ocaña en chef de files). Le journaliste relate un exploit dans sa récupération à une étape de la Vuelta et parle d'《 expérience» pour se référer aux soins médicaux :

«L'histoire, beaucoup de supporters la connaissent : moralement détruit, physiquement abattu et sans autre objectif que d'abandonner la course pour se rétablir de la très forte bronchite dont il souffrait, Agustín [Tamames] appela le docteur Gorospe à son hôtel de Bilbao. Ce dernier le soumit à deux sessions de relaxation sophronique avant d'affronter l'étape du lendemain, BilbaoMiranda de l'Ebre, avec en plus le col de l'Orduña à grimper, et... le miracle se fit. Tamames renaquit de ses cendres psychologiques et physiques de la veille. Non seulement il brilla tout au long de la course mais en plus il gagna le titre. Et pour couronner le tout, le jour suivant il répéta l'expérience et Tamames gagna de nouveau ${ }^{18}$.»

Sur la même ligne, nous trouvons une autre nouvelle dans laquelle est décrit le rapide rétablissement d'un autre cycliste espagnol, Javier Elorriaga, après une chute :

«Le rétablissement de Javier Elorriaga après l'accident grave qu'il a subi en compagnie d'Andres Gandarias a étonné tout le monde. Le coureur a commencé à monter sur son vélo bien que pour l'instant il ne puisse pas se lever de la selle pour monter les côtes. Les médecins sont tout à fait satisfaits, et après les soins intensifs auxquels se soumet le coureur, on espère qu'il puisse réapparaître dans les épreuves que le Kas a programmées sur la Côte d'Azur ${ }^{19}$. »

Finalement, il y a peu de cas où l'athlète a fait prévaloir un critère de protection de sa santé face au dopage. De ce qui ressort des chroniques, personne, encore moins les sportifs eux-mêmes, ne peut ignorer l'existence et la généralisation de ces pratiques ni leur danger, mais au final, d'autres intérêts l'emportent. Même chez le journaliste cette tendance saute aux yeux, ce qui démontre un aveuglement où intérêt pour défendre la prouesse sportive au risque de la santé du sportif.

Dans la relation entre corps, dopage et santé, une figure clé est l'équipe médicale. De façon constante, le sportif fait allusion à sa responsabilité dans l'affaire, alléguant qu'il a seulement pris ce qu'on lui a recommandé. En ce sens, nous trouvons plusieurs exemples. Sur le terrain, les personnes interviewées apportent des points de vue divers par rapport au type de produits utilisés pour la santé (allergies), pour récupérer et vitamines. Ces produits apparaissent comme des produits légaux et nécessaires pour la

\footnotetext{
18 Rufo S., « Regresa un emigrante de postín, Tamames », As, 12/01/1975, p. 18 .

19 Asombrosa recuperación de Elorriaga, Marca, 12/01/1975, p 21.
}

pratique du sport. Les changements de l'UCI pour supprimer la récupération intraveineuse par des cachets sont critiqués :

«Mais à l'UCI, ils ne se comprennent même pas entre eux, car je crois que pour récupérer comme ils le faisaient avec une injection intraveineuse ou autre chose, bien que cela soit un peu mauvais, c'était bien meilleur pour l'organisme parce que... maintenant en récupérant avec des cachets, peut-être qu'il faut en prendre vingt par jour. A long terme, l'organisme en souffre » (homme, cyclisme, 22 ans, Espagnol).

Nous pouvons aussi observer cette tendance d'un point de vue historique. Le premier d'entre eux est Bartolomé Caldentey qui a gagné une médaille de bronze au championnat du monde de cyclisme sur piste amateur en 1980 mais il a été contrôlé positif lors de l'épreuve. Sa réaction fut de se disculper et d'affirmer qu'il ne faisait que suivre les instructions du médecin et qu'il ne savait pas pourquoi dans certaines épreuves il était contrôlé positif et que dans d'autres il ne l'était pas :

«Quelques jours auparavant, aux championnats d'Espagne, disputés à Algaida, explique Caldentey, j'avais pris le complexe vitaminique que m'avait prescrit un médecin majorquin qui me dit que les suites du possible doping seraient déjà passées au moment du championnat du monde. . Je l'ai naturellement dit au médecin de la Fédération et celui-ci a calculé la date à laquelle ils m'injectèrent ce complexe et a considéré que c'était impossible d'être contrôlé positif. Quand je lui ai commenté cela, le sélectionneur national, Vidaurreta, était avec nous et lui aussi était d'accord [...]. Je ne sais pas ce qu'il se passe mais ce qui est certain c'est que certaines substances qui dans notre pays entraînent un contrôle négatif, dans des courses contrôlées par l'UCI entraînent un positif ${ }^{20}$. »

Également, dans l'affaire de Perico Delgado, gagnant du Tour de France 1988 et qui a été contrôlé positif au probenecid, la défense tant du coureur que celle de son équipe s'est concentrée sur l'affirmation que ce produit a été utilisé uniquement à des fins médicales :

«La fonction qu'il a est l'excrétion rénale. C'està-dire, retenir au maximum l'élimination de produits par l'urine du sportif. Face à ce fait, il a été décidé d'interdire son utilisation par les sportifs puisqu'il dissimule, en général, l'utilisation de substances dopantes. Toutefois, nous reconnaissons aussi le fait qu'il serve de régulateur de l'acide

20 Avespa J., «Caldentey, desmoralizado, anuncia su retirada : '...A no ser que se me reconozca públicamente que no me he dopado' 'tomé un complejo vitamínico que me recetó un médico mallorquín'», Marca, 28/09/1980, p. 21. 
urique, fonction pour laquelle Perico Delgado l'a utilisé ces dernières années ${ }^{21}$.»

Les conclusions du travail Guerreschi et Garnier (Guerreschi \& Garnier, 2008, p. 239-294) sur les représentations du dopage sportif montrent qu'il est difficile de concevoir sans ambiguïté une limite entre ce que représenterait et ne représenterait pas le dopage : «Même quand les athlètes essaient d'établir une limite entre dopage et aide énergétique, l'aspect moral ressort toujours. Se doper, c'est l'intention de tricher. [...] D'une certaine façon, c'est l'intention qui compte ${ }^{22}$.

D'autre part, nous ne trouvons pas de déclarations chez les sportifs qui s'accusent les uns les autres soupçonnant un possible dopage. L'unique cas trouvé où un sportif met en évidence un possible dopage en raison de signes physiques ou externes d'autres sportifs est le fait du cycliste Sebastián Pozo, coureur d'Âlava de l'équipe Kas. L'affaire Pozo eut lieu pendant le Tour de France de 1977. Le cycliste fut contrôlé positif lors de la dixneuvième étape. La sanction fut de quatre mois ce qui eu des répercussions négatives sur l'équipe du Kas qui perdit le leadership du classement par équipes. Pozo était récidiviste puisque dans une précédente épreuve il ne s'était pas présenté au contrôle antidoping et il fut suspendu pendant un mois. Quelques mois plus tard, après avoir été contrôlé positif, Mundo Deportivo décrivait la sanction de Pozo comme « un verdict polémique ». Quand ce dernier était accusé de dopage et finalement sanctionné, le coureur soupçonnait d'autres cyclistes :

«Ce qui est certain c'est que Pozo nous parle indigné. Et à juste titre. Il s'est ensuite étendu sur des considérations sur certains coureurs qui apparaissent le matin avec le visage gonflé non pas de sommeil. Il commentait aussi que la "mafia" des "grands" avait commencé à fonctionner et que cela continuera à exister tant que tous les hommes qui régissent le cyclisme international ne seront pas changés ${ }^{23} \gg$.

\section{II existe un dopage, oui, mais ce sont toujours «les autres »}

Quand nous parlons de la représentation du dopage dans les cultures sportives analysées (athlétisme, cyclisme et basketball), celle-ci est inégale. Le cyclisme apparaît comme le sport le plus sanctionné en nombre de contrôles positifs, politique antidopage et d'influence de la presse en tant que juge et partie dans les affaires, suivi de l'athlétisme et finalement du basketball avec des affaires anecdotiques et jamais d'origine espagnole.

\footnotetext{
21 Esteban M., «Un positivo más que dudoso », Marca, $21 / 07 / 1988$, p. 5 .

22 Ibid, 288.

23 Carrasco J., «Pozo protestaba », Marca, 10/07/1977, p. 22 .
}

Dans n'importe quel des trois sports, la position face au dopage est toujours celle de la négation, et c'est un discours constant pendant les 35 années analysées. Y compris en ayant évolué d'un discours d'ingénuité dans la première étape (années 1970 et 1980) jusqu'à l'étape la plus récente où la connaissance des substances et des méthodes dopantes de pointe est beaucoup plus étendue tant chez les sportifs que chez le public en général. Le discours chez l'athlète soupçonné est toujours le même : la négation du dopage et la possibilité de responsabiliser les autorités sportives et antidopage pour le harcèlement exercé envers le sportif, comme dénoncé de manière constante en cyclisme en ce qui concerne l'UCI. Par exemple, Llaneras en 2001 accuse l'UCI d'être le seul organisme sportif à expérimenter des méthodes de détection pas encore contrastées, avec le risque que cela comporte d'accuser des personnes de contrôles positifs qui en réalité ne le sont pas ${ }^{24}$. Cependant, cette position anti-UCI des athlètes espagnols n'a pas toujours été la même, surtout lors de l'étape préalable à la création de l'AMA : dans l'affaire Perico Delgado (1988), le président du plus grand organisme cycliste à ce moment-là, l'Espagnol Luis Puig, est alors intervenu personnellement dans l'affaire pour défendre face à l'organisation du Tour que la substance qui avait été contrôlée positive n'était pas dans la liste de l'UCI et, par conséquent, cette défense permit à Perico de gagner le tour gaulois. Dans cette affaire, la presse a fait attention d'utiliser constamment l'argument, en évoquant que l'UCI était la plus grande autorité et que sa liste de substances « est celle qui régit toutes les $\operatorname{courses}^{25} \gg$.

Nous avons trouvé un seul cas de reconnaissance publique de dopage, et seulement quand le contrôle positif a été rendu public par la presse et non motu proprio. Il s'agit de l'affaire Marga Fullana, cycliste de mountain bike dans version cross-country titulaire d'un vaste palmarès : championne du monde, championne d'Europe et médaille de bronze à Sydney en 2000. Au championnat du monde de 2010, le contrôle positif de Marga Fullana à l'EPO fut annoncé et une sanction de 2 ans sans pouvoir concourir fut confirmée. L'affaire Fullana est unique pour ne pas avoir trouvé d'antécédents de confession de culpabilité chez des sportifs espagnols jusqu'à présent, ainsi que l'honnêteté dans ses déclarations, au moment où était rendu public le contrôle positif au clenbuterol d'Alberto Contador au Tour de France. Les raisons que la cycliste énuméra furent associées au faible rendement :

«J'ai commis la stupidité la plus grande de ma vie. J'ai eu une très mauvaise année, physiquement et mentalement. Je ne retrouvais mes sensations dans aucune course. Je me mettais la pression. On me mettait la pression. À cela, est venu s'ajouter des problèmes personnels et plusieurs lésions

\footnotetext{
24 Torelló R., «Llaneras : 'no soy un drogadicto'», Mundo Deportivo, 27/11/2001, p. 30.

25 Dalmases J. «La mano izquierda de Luis Puig », Mundo Deportivo, 22/07/1988, p. 5.
} 
consécutives [...]. Je n'ai jamais eu recours au dopage avant. Je n'en avais pas besoin mais cette année je n'arrivais à rien et j'ai eu la maudite opportunité d'obtenir cette substance [EPO], mais une quantité tellement petite que je n'ai rien remarqué dans mon rendement. Je n'ai rien amélioré et le championnat du monde s'est déroulé comme toutes les autres courses : $\mathrm{mal}^{26} \gg$.

Fullana assuma toute la faute sans responsabiliser le fournisseur de l'EPO bien qu'elle considéra peu éthique d'apprendre son dopage par la presse et non par l'UCI. Malgré l'importance de cette affaire, son impact fut mineur, car n'affecta pas une star du sport ni une discipline de grande répercussion comme l'est le cyclisme féminin. La cycliste, malgré la sanction, reçut un hommage dans un club de Barcelone quelques mois après, avec d'autres sportifs majorquins $^{27,28}$. À son retour à la compétition en 2013, dans aucun cas il n'a été fait mention de sa sanction pour dopage au contraire de ses mérites sportifs obtenus précédemment ${ }^{29}$.

\section{Le cyclisme, la « brebis galeuse » du sport}

Comme nous le commentions au début, en abordant la représentation construite sur le dopage dans les trois sports analysés, il est intéressant de faire une mention spéciale à la grande différence entre les sports, la culture cycliste étant la plus touchée en ce sens. Dans les deux périodes historiques analysées, les cyclistes, les entraîneurs, les organisateurs des épreuves et les dirigeants de fédérations maintiennent un discours constant dans le temps dans lequel le cyclisme est poursuivi de façon beaucoup plus véhémente par rapport à d'autres sports, et s'auto-considère la « brebis galeuse » ou la « tête de turc » du sport mondial quant au dopage.

D'un point de vue quantitatif, ce désajustement peut être observé autour du volume de nouvelles sur le dopage. Dans leur majorité, il s'agit du cyclisme bien que nous trouvons des articles qui traitent de cas dans d'autres disciplines sportives, comme l'athlétisme, la boxe, le football, ou l'automobilisme mais de façon ponctuelle. Pour illustrer cette situation, nous pouvons prendre comme exemple l'année 1975 dans le quotidien Marca : sur les 66 nouvelles analysées qui traitent le sujet du dopage directement ou indirectement, le cyclisme représente 31 articles, c'est-à-dire $47 \%$ du total. Face à cela, l'athlétisme

\footnotetext{
26 Pons P., « Fullana admite que se dopaba », Mundo Deportivo, 02/10/2010, p. 37.

27 Sanchís A., « Hoy, Gala de Campeones y VIII Aniversario de la Penya Els Tamarells en Mallorca », Mundo Deportivo, 10/12/2010, p. 16.

28 Gala dels Campions- $8^{\text {ème }}$ aniversari P.B. Els Tamarells. http://www.penyabarcelonistaelstamarells.cat/archives/

date/2010/12, page consult \{ee le 15/11/2014.

29 « Marga Fullana vuelve a competir », Mundo Deportivo, 02/06/2013, p. 42.
}

compte 8 nouvelles, le football et l'équitation 6 , la boxe 5 , et le judo, la natation et le waterpolo 2. Cette prédominance de nouvelles sur le dopage dans le cyclisme peut être comprise par les plus grandes exigences de contrôles permanents et quotidiens si nous le comparons aux autres disciplines sportives ; fait dont se feront l'écho les journalistes en défense de l'athlète « poursuivi ». Toutefois, à cette époque, nous avons aussi trouvé des cas de dopage dans d'autres sports, avec des apparitions dans le journal non négligeables, comme par exemple en Formule $1^{30}$ ou en football ${ }^{31}$.

Précisément, c'est dans « le sport roi $»$ en Espagne que l'on va donner une couverture médiatique très abondante quand finalement il est décidé de mettre en œuvre des contrôles antidopage en 1988, juste après le scandale des Jeux de Séoul et le contrôle positif de Ben Johnson; même le football sera érigé, à tort par les journalistes, de sport «pionnier » dans la lutte contre le dopage ${ }^{32}$. Mais la domination du football sur d'autres sports comme le cyclisme aura lieu aussi des années avant le Tour d'Espagne en 1982, quand Arroyo, vainqueur de l'épreuve, fut disqualifié. Comme publia Mundo Deportivo, sur le Tour d'Espagne, les machines de détection de substances dopantes furent étrennées pour les essayer pour la coupe du monde de football en Espagne en 1982, qui aura lieu seulement un mois après :

«Le second détail significatif que nous pouvons ti-
rer comme conclusion de toute cette "affaire" est
qu'une fois de plus les cyclistes, comme dit Ar-
royo lui-même, ne sont pas plus que les têtes de
turc d'un fléau du sport en général. Des machines
perfectionnées ou étrennées à l'occasion du Tour
d'Espagne pour "entrâ̂ner" leurs services pour la
coupe du monde de football ont été celles qui ont
considéré Arroyo coupable 33 . »

Nous trouvons un exemple plus récent en 2001 dans l'affaire Joan Llaneras, cinq fois champion du monde de cyclisme sur piste et détenteur de quatre médailles olympiques (Sydney 2000, Atlanta 2004 et Pékin 2008), qui a

\footnotetext{
30 Ventura X., «Doping y automovilismo, el 'caso Stuck', claro ejemplo de una vieja historia », Mundo Deportivo, 15/04/1977, p. 23.

31 Concrètement, le rapport que Marca consacra en octobre 1988 au programme anti dopage dans la Ligue Espagnol de Football (LFP) n'a pas de précédents dans d'autres disciplines sportives : deux jours de suite, avec un reportage exhaustif (samedi et dimanche) à double page, avec l'explication détaillée de toutes les substances, y compris à usage domestique, qui seraient contrôlées positives, les pas à suivre lors d'un contrôle, accompagné de colonnes d'opinions médicales et psychologiques. Esteban M. et Montero A., «Informe MARCA : todo sobre el control antidoping en el fútbol español », Marca, 09/10/1988, p. 14-15.

32 Esteban M. et Montero A., «Informe MARCA : así se castigará el dóping », Marca, 08/10/1988, p. 18.

33 Dalmases J., «Definitivo : Arroyo descalificado en la Vuelta a Espaãa », Mundo Deportivo, 14/05/1982, p. 31.
} 
été inculpé d'être contrôlé positif à l'EPO, mais son cas a finalement été annulé en raison d'erreurs dans la méthode de détection de la substance. Lors de la conférence de presse qui a suivi la publication de son affaire, Llaneras a mentionné le cas du footballeur Pep Guardiola qui, à ces mêmes dates, a été contrôlé positif à la nandrolone quand il jouait dans l'équipe Brescia de la ligue italienne Série A :

«Ce ne sont pas des situations comparables, car le football fait bouger une grande quantité de médias. Les titulaires, les manifestations et le traitement sont différents. Il est difficile que dans la presse apparaissent des affirmations aussi dures qu'elles l'ont été contre ma personne. Cependant, je sais ce par quoi Guardiola doit être en train de passer en ce moment ${ }^{34}$. »

Nous trouvons le cas le plus récent dans l'Opération Puerto où Eufemiano Fuentes est à nouveau le protagoniste dans une affaire de dopage - et ici, le principal impliqué -, confirmant dans les médias comme dans l'interview que lui a fait $L e$ Monde $^{35}$, que dans son portefeuille de 《clients » habituels qui se soumettent à des traitements dopants non seulement figuraient des cyclistes mais aussi des footballeurs, joueurs de tennis, athlètes, boxeurs et autres sportifs. Le docteur Fuentes a assuré que l'anonymat du reste des sportifs, surtout des footballeurs (première et seconde division de la ligue professionnelle) est dû au fait que certains sports ont davantage de pouvoir que d'autres, et que de publier les noms entraînerait la destitution du plus haut dirigeant politique sportif, le Secrétaire d'État chargé des sports à ce moment-là, Jaime Lissavetzky.

Sur le terrain, le football est le sport pour lequel les jeunes sportifs ont le plus de soupçons en raison de l'opacité dans les données sur le dopage existant dans le sport le plus médiatique d'Espagne, ainsi que par l'existence d'un double critère pour certains sports :

«C'est un sujet qu'il faut contrôler, mais ces derniers temps non seulement nous le voyons beaucoup en cyclisme, en athlétisme, mais par exemple en football on ne voit rien, tu sais? Qu'est-ce que j'en sais? Peut-être parce que c'est le sport le plus important en Espagne, peut-être que ça n'a rien à voir mais, je ne sais pas. Oui, je crois qu'il faut faire des contrôles parce que, je ne sais pas, le sport doit être sain et... je ne sais pas, se doper c'est

34 Torelló R., «Llaneras : 'no soy un drogadicto'», op. cit., p. 31 .

35 Mandard S., «Le docteur Eufemiano Fuentes, organisateur présumé d'un réseau de dopage, affirme qu'il a eu des 'athlètes, joueurs de tennis, footballeurs' comme clients 'On m'a menacé de mort trois fois'», Le Monde, 08/12/2006, http://www.lemonde.fr/cgi-bin/ACHATS/acheter.cgi? offre $=\mathrm{ARCHIVES} \&$ type _item $=\mathrm{ART}$ _ARCH_30J\&objet $\mathrm{id}=968809 \& \mathrm{xtmc}=$ eufemiano fuentes $\& \mathrm{xtcr}=\overline{11} 6$, page consultée le 15/11/2013 déjà tricher, avec les gens qui ne... je ne sais pas» (femme, athlétisme, 20 ans, Espagnole).

«Oui. Il se peut qu'il y ait une certaine tolérance. Ce qui se passe c'est que ce n'est pas la même tolérance dans tous les sports non plus. Alors, la tolérance ça dépend pour quoi. En cyclisme, tout le monde dit ah, ils se shootent énormément, mais réellement dans le football peut-être aussi et ce n'est pas aussi étudié. Alors, tout est un peu irrégulier non? » (femme, athlétisme, 21 ans, Espagnole).

Le traitement inégal du cyclisme par rapport à d'autres sports découle non seulement de la plus grande ou plus petite influence économique ou médiatique des uns sur les autres mais aussi des autorités politiques sportives ellesmêmes. Un exemple clair, nous le trouvons dans la nouvelle de Marca, dans sa dernière page du 8 décembre 1985, avec le titre « Le doping ravage le sport espagnol ${ }^{36} \gg \mathrm{pu}-$ bliant en exclusivité un rapport officiel du Laboratoire de recherche biochimique et de contrôle du doping CSD, le Conseil Supérieur des Sports, organisme politique sportif le plus important en Espagne. Dans ce rapport, on informe d'un total de 301 contrôles positifs comptabilisés entre 1969 et 1984 dans différentes disciplines sportives, le cyclisme possédant le plus grand nombre de contrôles positifs (251 sur 6757 prélèvements analysés). Toutefois, la boxe ( 7 contrôles positifs sur 53 prélèvements) et l'haltérophilie (17 sur 287) dépassaient le cyclisme en pourcentage de cas. En athlétisme, par exemple, on a comptabilisé 17 contrôles positifs qui n'ont jamais vu le jour et dont on n'a jamais connu les noms.

La perception des jeunes cyclistes que leur sport ait été le plus touché par rapport au reste des disciplines est semblable à la tendance dans les cas trouvés dans les journaux :

«Bon, c'est complètement erroné parce que, comme dans d'autres sports, on donne toujours le maximum et tout. Et... s'il est vrai que le cyclisme est gravement touché par le truc des substances dopantes, mais c'est un... c'est quelque chose qui est venu maintenant, avant... par exemple, peut-être que d'autres sports ont été touchés par le dopage aussi, car maintenant... c'est toujours le tour de quelqu'un. Maintenant, c'est le tour du cyclisme, une autre année ce sera celui de l'athlétisme, une autre année tel. . toujours... (homme, cyclisme, 14 ans, Espagnol).

Un cas exceptionnel d'une plus grande répercussion médiatique en cyclisme par rapport à d'autres sports, non tant pour le sport en soi mais pour l'importance individuelle du sportif, nous pouvons l'observer chez l'athlète espagnol Alberto García Fernández. Médaille d'or au championnat d'Europe de 2002, aux 5000 mètres, et $3^{\mathrm{e}}$ au championnat du monde, ainsi que Prix National des

\footnotetext{
36 Molero P., «El doping asola al deporte español », Marca, 08/12/1985, dernière page.
} 
Sports de cette même année. Le 10 octobre 2003, García confirma la sanction de deux années de suspension après avoir été déclaré coupable d' « ingestion exogène» d'EPO. L'athlète le nia car il considérait qu'il s'agissait d'un faux positif mais ne retournera pas sur la piste jusqu'au 9 juin 2005. Cependant, à son retour il ne réussit pas à atteindre à nouveau son niveau d'avant l'affaire de dopage. L'athlète ne fit pas appel de la sentence par manque de moyens économiques et de soutien politique :

\section{«En ce qui concerne ses possibilités de défense [Alberto García], il a dit que c'est une lutte d'une personne avec une capacité économique limitée. Si j'avais pu engager une équipe d'avocats et de bio- chimistes, je ne sais ce qui aurait pu se passer. $C^{\prime}$ 'est le doute que j'aurai toujours ${ }^{37}$. »}

Si nous le comparons à l'affaire Contador, par exemple, nous pouvons observer le traitement inégal quant à la capacité qu'a eu le cycliste pour mobiliser en sa faveur le traitement politique et institutionnel de plus haut rang, ainsi que de disposer d'une importante équipe d'avocats qui permit de faire appel pour développer au maximum un long processus judiciaire devant le Tribunal Arbitral du Sport (TAS). Dans le cas de Contador, la citation suivante illustre cette idée :

«[...] Son problème [en référence à Contador] est le fruit des luttes politiques incessantes entre l'Agence Mondiale Antidopage (AMA) et l'UCI d'une part et les autorités espagnoles d'autre part [...]. L'UCI a toujours accusé le cyclisme espagnol d'être excessivement complaisant avec les personnes qui se dopent ${ }^{38}$. »

Sous ces accusations de l'UCI, différentes autorités politiques espagnoles comme le Secrétaire d'État chargé des Sports à ce moment-là, Jaime Lissavetzky, l'agence publique antidopage et le Conseil Supérieur des Sports défendirent la politique antidopage qui est réalisée en déclarant que «l'Espagne n'a pas de problème spécial avec le dopage $^{39}$ », ou que l'Espagne est située « à l'avant-garde de la lutte contre le dopage $e^{40} »$. Cependant, les déclarations de Lissavetzky dénotent une préoccupation pour l'image internationale qui est donnée de l'Espagne : «il n'y a pas un problème ni plus grand ni plus petit que dans le reste du monde » avec le dopage bien qu'il ait reconnu que la supposée implication de sportifs de premier rang affecte l'image ${ }^{41}$. De l'Association de Sportifs Professionnels, on accuse aussi l'AMA en disant que : «L'AMA a été créé à la demande des gouvernements mais maintenant elle paraît être au-dessus du bien et du mal. Nous n'avons pas de droit de vote ni de voix et ils nous voient

\footnotetext{
37 Alberto Garcia : «mi ejemplo es Julio Rey», Mundo Deportivo, 09/10/2003, p. 31.

38 El País, 10/11/2010, p. 55.

39 El País, $17 / 11 / 2010$, p. 47.

40 El Mundo, $12 / 12 / 2010$, p. 43.

41 El Mundo, 08/10/2012, p. 50.
}

comme les méchants du film ${ }^{42} »$, en indiquant qu'effectivement au sein de cet organisme international les sportifs n'ont pas de représentation.

Ainsi, Contador reçut l'appui de nombreuses figures publiques qui ont pris sa défense, parmi elles le président du Comité Olympique Espagnol (COE), Alejandro Blanco ${ }^{43}$, le leader de l'opposition (Parti Populaire) à ce moment-là, Mariano Rajoy ${ }^{44}$ et même le président du Gouvernement, José Luis Rodriguez Zapatero qui déclara sur Twitter : «Il n'y a aucune raison juridique pour sanctionner Contador ${ }^{45}$.»

Remerciements. Cet article s'inscrit dans le cadre du projet de recherche «Prévenir le dopage chez les jeunes sportifs en France et en Espagne », financé par l'Agence Mondiale Antidopage (AMA,) à travers du Social Science Research Grant Program 2010.

\section{Bibliographie}

Chartier C. (2011). Les enjeux de la nouvelle presse économique dans l'Espagne des années 1980, Cahiers de civilisation espagnole contemporaine, 8.

Dimeo, P. (2007). A history of drug use in sport 1876-1976. Beyond good and evil. Londres : Routledge.

González Aja T. (2011). "Contamos contigo" : sociedad, vida cotidiana y deporte en los años de desarrollismo, 1961-1975. In X. Pujadas i Martí (Dir.), Atletas y ciudadanos. Historia social del deporte en España 1870-2010 (pp. 323-353). Madrid : Alianza Editorial.

Guerreschi L., \& Garnier C. (2008). Les représentations sociales du dopage sportif. Étude qualitative auprès d'athlètes de haut niveau français et canadiens. Drogues, Santé et Société, 7 (1), 239-294.

López López B. (2011). El impacto social y cultural del deporte en la España del bienestar: televisión, consumo y deporte mediático, 1982-2000. In X. Pujadas i Martí (Dir.), Atletas y ciudadanos. Historia social del deporte en España 1870-2010 (pp 393-432). Madrid : Alianza Editorial.

Møller, V. (2012). Un diablo llamado dopaje. Cultura Ciclista : Tarragona.

Perera E. \& Gleyse J. (2005). Le dopage dans quatre grands périodiques sportifs français de 1903 aux années soixante. Le secret, le pur et l'impur, STAPS, 70, 89-108.

Pujadas i Martí X. \& Santacana C. (1997). L'esport és noticia : història de la premsa esportiva a Catalunya (1880-1992) Barcelona : Diputació de Barcelona-Collegi de Periodistes de Catalunya.

Viuda Serrano A. (2010). El atleta de la Posguerra Civil Española. La transmisión de los valores franquistas a través de la censura. In J. Sampedro Molinuevo, T. González Aja et N. García-Arjona (Dir.), El atleta olímpico:?'transmisor de valores? Una aproximación histórico-científica (pp. 157-176). Madrid : Atos Origin.

\footnotetext{
42 El Mundo, 08/10/2012, p. 50.

43 El Mundo, 28/10/2010, 53.

44 El Mundo, 14/02/2011, 13.

45 El País, 11/02/2011, dernière page.
} 